\title{
The GATE summer school
}

\author{
M. Skarka ${ }^{1,2}$, J. Janík ${ }^{2}$, E. Paunzen ${ }^{2}$ and V. Glos ${ }^{2}$ \\ 1 Astronomical Institute of the Czech Academy of Sciences, Fričova 298, \\ CZ-25165 Ondřejov, Czech Republic (E-mail: skarka@asu.cas.cz) \\ 2 Department of Theoretical Physics and Astrophycis, Masaryk University, \\ Kotlářská 2, CZ-61137 Brno, Czech Republic
}

Received: November 25, 2020; Accepted: November 26, 2020

\begin{abstract}
The education activities in the form of short and condensed courses such as summer schools are essential parts of the educational process of every scientist. Here we briefly describe the program and the organization of the GATE summer school that was held in a form of virtual meeting between August 8 and 15 in Brno, Czech Republic.
\end{abstract}

Key words: GATE summer school - General: editorial - Methods: data analysis

\section{Introduction}

In the time of large sky surveys and projects producing large amount of data, it is very important to know where to find the archived and freely available data, how to work with them and what tools and pipelines to use to work efficiently. However, without the knowledge of the physics underlying the studied phenomena, working with the data would be useless. The two recent most important satellites regarding the stellar astrophysics are GAIA (Gaia Collaboration et al., 2018) and TESS (Ricker et al., 2014). Our motivation to organize a summer school dedicated to these two missions was to provide the students with knowledge about the usage of the data produced by these satellites in a short time of the summer school.

We named the school "GAIA \& TESS: Tools for understanding the Local Universe", shortly GATE summer school ${ }^{1}$. It was foreseen as the second summer school under the ERASMUS+ project titled "Per Aspera ad Astra Simul" that secures mobility of students, teachers and researchers among institutes in the Czech Republic, Slovak Republic, and Canarias, Spain (Kabáth et al., 2019). The first summer school was organized in cooperation with the OPTICON consortium and was held in Stará Lesná in Slovak Republic at the AI SAV between 17 and 27 July, 2019 (Kabáth et al., 2019).

\footnotetext{
${ }^{1}$ https://gate.physics.muni.cz/
} 


\section{The organization of the school}

The GATE summer school was organized by the Department of Theoretical Physics and Astrophysics, Masaryk university in Brno, Czech Republic (the main organizer) in cooperation with the Astronomical institute, AS CAS, Ondřjov, Czech Republic. Originally, the school was proposed for 30 students across the world working on six different topics in groups comprising five students under supervision of experienced scientists. The original date of the school (June 2020) had to be postponed to August due to the COVID-19 pandemic. This postponement resulted in the reduction to four projects due to the unavailability of tutors. In addition, 14 days before the start of the school, we were forced to go in a fully online regime because the in-person format was impossible to manage.

During July 2020, 20 students $^{2}$ from seven different countries were selected to participate on the school in the new dates of August 8 to 15. Finally, only 17 students of BSc to $\mathrm{PhD}$ stages were able to participate on the school. About 40 hours were dedicated to the work on four projects from the stellar astrophysics: Exoplanets (lead by Hannu Parviainen, IAC Tenerife, Spain), Stellar pulsations (lead by Paul Beck, University of Graz, Austria), Eclipsing binaries (lead by Kyle Conroy, Villanova University, United States) and Stellar flares (lead by Krisztian Vida, Konkoly Observatory, Hungary). The rest of the time was filled with lectures about the TESS and GAIA missions, as well as about Kepler (Borucki et al., 2010) and BRITE satellites (Weiss et al., 2014). There were also two hands-on sessions on TESS and GAIA data and a career session.

All the communication and lectures were online using Zoom and Slack. After the initial get in use, these tools appeared to be very efficient and easy to use. The lectures were recorded and are available at Youtube. $\mathrm{com}^{3}$, the presentations are posted at the web page of the summer school. Despite the unpleasant situation, we managed to meet online at the conference party and have two hours of fun. The participants including the tutors are in Fig. 1.

\section{The projects}

The projects and the results are described in this CAOSP issue. Briefly, the exoplanet group revised the parameters of four transiting hot Jupiter planets by using PyTransit software (Parviainen, 2015) on the TESS data (Gajdoš et al., 2021). The binary group tested the robustness and accuracy of parameters using the soon to be released PHOEBE 2.3 EB modeling package (Conroy et al., 2020) on the TESS data of AI Phe (Korth et al., 2021). Project on stellar flares was focused on search for flare stars in 136 open clusters by using TESS light curves and utilizing GAIA data (Maryeva et al., 2021). Finally, the asteroseismic group

\footnotetext{
2 from 51 registered applicants

${ }^{3}$ https: //www . youtube.com/channel/UCHk-EY_tFtGKotKn9xt3PeA
} 


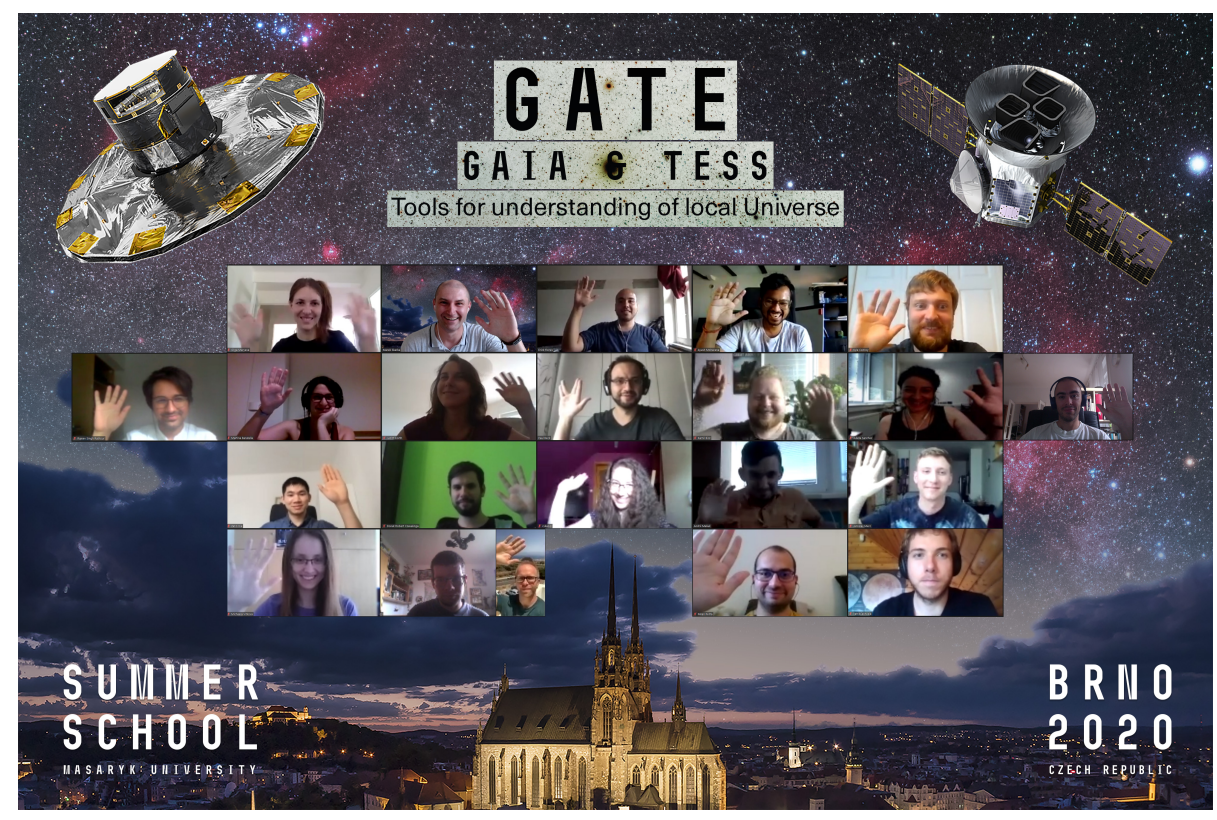

Figure 1. The conference photo of all participants of the GATE summer school including the main organizer and tutors.

worked on the global asteroseismic analysis of a heart-beat star KIC 5006817 based on the full Kepler data sample and TESS data (Merc et al., 2021).

\section{Summary}

We briefly summarized the GATE summer school devoted to TESS and GAIA missions held in August 8-15 in Brno, Czech Republic. The school was held in fully online regime. The students attended six lectures focused on the space missions, two hands-on sessions focused on TESS and GAIA data, one career session, and participated on an online version of a conference party. The lectures are available online on the web page of the summer school and on Youtube.com. Overall, the students, tutors and lecturers did a great job. The results of the students projects are summarized in (Gajdoš et al., 2021; Maryeva et al., 2021; Korth et al., 2021; Merc et al., 2021, this proceedings).

Acknowledgements. We would like to thank all the people involved in the summer school. Special thanks goes to the main coordinator of ERASMUS+ project, Petr Kabáth, who helped with the practical issues. The summer school was funded from the ERASMUS+ grant number 2017-1-CZ01-KA203-035562. MS acknowledges the Opera- 
tional Program Research, Development and Education - Project Postdoc@MUNI (No. CZ.02.2.69/0.0/0.0/16_027/0008360) and the MŠMT grant LTT20015.

\section{References}

Borucki, W. J., Koch, D., Basri, G., et al., Kepler Planet-Detection Mission: Introduction and First Results. 2010, Science, 327, 977, DOI: 10.1126/science.1185402

Conroy, K. E., Kochoska, A., Hey, D., et al., Physics of Eclipsing Binaries. V. General Framework for Solving the Inverse Problem. 2020, Astrophysical Journal, Supplement, 250, 34, DOI: 10.3847/1538-4365/abb4e2

Gaia Collaboration, Brown, A. G. A., Vallenari, A., et al., Gaia Data Release 2. Summary of the contents and survey properties. 2018, Astronomy and Astrophysics, 616, A1, DOI: 10.1051/0004-6361/201833051

Gajdoš, P., Maliuk, A., Vítková, M., \& Parviainen, H., Improving light curve parameters of exoplanets based on TESS data. 2021, Contrib. Astron. Obs. Skalnaté Pleso, 51, 68, DOI: $10.31577 /$ caosp.2021.51.1.68

Kabáth, P., Korhonen, H., \& Jones, D., Observational astrophysics: from proposals to publication. 2019, Contributions of the Astronomical Observatory Skalnate Pleso, 49, 522

Korth, J., Moharana, A., Pešta, M., Czavalinga, D. R., \& Conroy, K. E., Consequences of parameterization choice on eclipsing binary light curve solutions. 2021, Contrib. Astron. Obs. Skalnaté Pleso, 51, 58, DOI: 10.31577/caosp.2021.51.1.58

Maryeva, O., Bicz, K., Caiyun, X., et al., Flare stars in nearby Galactic open clusters based on TESS data. 2021, Contrib. Astron. Obs. Skalnaté Pleso, 51, 78, DOI: 10.31577/caosp.2021.51.1.78

Merc, J., Kalup, C., Rathour, R., Sánchez Arias, J., \& Beck, P., Asteroseismology of the heartbeat star KIC 5006817. 2021, Contrib. Astron. Obs. Skalnaté Pleso, 51, 45, DOI: $10.31577 /$ caosp.2021.51.1.45

Parviainen, H., PYTRANSIT: fast and easy exoplanet transit modelling in PYTHON. 2015, Monthly Notices of the RAS, 450, 3233, DOI: 10.1093/mnras/stv894

Ricker, G. R., Winn, J. N., Vanderspek, R., et al., Transiting Exoplanet Survey Satellite (TESS). 2014, in Society of Photo-Optical Instrumentation Engineers (SPIE) Conference Series, Vol. 9143, Space Telescopes and Instrumentation 2014: Optical, Infrared, and Millimeter Wave, ed. J. Oschmann, Jacobus M., M. Clampin, G. G. Fazio, \& H. A. MacEwen, 914320

Weiss, W. W., Rucinski, S. M., Moffat, A. F. J., et al., BRITE-Constellation: Nanosatellites for Precision Photometry of Bright Stars. 2014, Publications of the ASP, 126, 573, DOI: $10.1086 / 677236$ 